La revue La revue pour l'histoire du CNRS

POUR LHISTORE DU CNRS $\quad 20 \mid 2008$

Aventures et recherches aux pôles

Glaciers, calottes polaires, climat et environnement

Le LGGE fête ses 50 ans

Paul Duval et Véronique Roux

(2) OpenEdition

Journals

Édition électronique

URL : https://journals.openedition.org/histoire-cnrs/5862

DOI : $10.4000 /$ histoire-cnrs.5862

ISSN : 1955-2408

Éditeur

CNRS Éditions

Édition imprimée

Date de publication : 3 avril 2008

ISBN : 978-2-271-06562-9

ISSN : $1298-9800$

Référence électronique

Paul Duval et Véronique Roux, "Glaciers, calottes polaires, climat et environnement », La revue pour I'histoire du CNRS [En ligne], 20 | 2008, mis en ligne le 03 avril 2010, consulté le 20 mai 2021. URL :

http://journals.openedition.org/histoire-cnrs/5862 ; DOI : https://doi.org/10.4000/histoire-cnrs.5862

Ce document a été généré automatiquement le 20 mai 2021

Comité pour l'histoire du CNRS 


\title{
Glaciers, calottes polaires, climat et environnement
}

\author{
Le LGGE fête ses 50 ans
}

Paul Duval et Véronique Roux

1 À l'origine, les premiers travaux réalisés au LGGE ont porté sur l'étude des mécanismes de glissement et de fluctuations des glaciers tempérés, puis se sont rapidement tournés vers l'analyse des carottes de glace, fournissant des informations capitales sur le climat et la composition de l'atmosphère (aujourd'hui sur plus de 800000 ans). Ces études et l'expérience acquise sur le terrain ont permis d'élargir les domaines de compétence du LGGE à des recherches sur la modélisation du climat, la chimie de l'atmosphère, la physique et mécanique de la glace et la dynamique des calottes polaires et de la banquise arctique.

Du laboratoire de l'aiguille du Midi au LGGE

2 Au cours de son séjour au Chili, Louis Lliboutry fait partie de l'expédition française dirigée par Lionel Terray, parti en Argentine en 1952 pour gravir le Fitz Roy. C'est en restant au camp de base que L. Lliboutry pris sa décision de créer un laboratoire de glaciologie à Grenoble. Dès son retour en France en 1956, il développe des études sur le glissement des glaciers et obtient de Jean Coulomb, directeur général du CNRS, la direction d'un laboratoire propre du CNRS, à Chamonix, situé à plus de $3500 \mathrm{~m}$ d'altitude. Le laboratoire des rayons cosmiques du col du Midi devient alors le laboratoire de l'aiguille du Midi. Malgré son penchant pour de nouvelles théories, L. Lliboutry a vite saisi l'importance des techniques et des mesures de terrain. Le LGGE comprend alors quelques techniciens et ingénieurs et s'implante en 1961 dans les locaux de l'ancien évêché de Grenoble avec un atelier de mécanique et d'électronique et déjà une chambre froide. L'année 1970 est une date importante avec l'arrivée de Claude Lorius et d'une petite équipe alors sur une péniche à Saint- Cloud. Les recherches du Laboratoire de glaciologie alpine vont s'orienter vers les régions polaires et rapidement vers la reconstruction du climat et de la composition de l'atmosphère grâce à l'analyse des carottes de glace de l'Antarctique. Les techniques acquises pour les carottages seront une clé des succès futurs du LGGE. 
3 Le Laboratoire de glaciologie devient en 1978 le Laboratoire de glaciologie et géophysique de l'environnement (LGGE) et s'installe sur le campus en 1982. Claude Lorius en devient le directeur en 1984. Plusieurs chercheurs de l'Institut de recherche et développement (IRD) spécialisés dans l'analyse de la relation climat/bilan de masse des glaciers andins rejoignent le laboratoire en 2000. Le LGGE devient une unité mixte de recherche CNRS/Université Joseph-Fourier de Grenoble en 2002. Ce laboratoire de l'observatoire des sciences de l'Univers de Grenoble (OSUG) est actuellement dirigé par Michel Fily et Jérôme Chappellaz.

Des recherches fondamentales à fort impact sociétal

4 Il est toujours difficile de souligner à un instant donné quelques résultats parmi tous ceux obtenus durant ces 50 ans. Le recul est certainement insuffisant et l'impact sociétal des recherches menées par le LGGE peut fausser cette analyse. Aussi, nous limiterons-nous sans grande objectivité aux résultats qui ont marqué la communauté scientifique internationale et maintenant le grand public. Le rayonnement du LGGE au niveau international a commencé très vite avec les discussions toujours enflammées entre L. Lliboutry et ses collègues et/ou concurrents sur les mécanismes de glissement des glaciers et, en particulier, sur le rôle de l'eau sous-glaciaire. Les carottages réalisés dès 1968 sur les glaciers de Saint-Sorlin (massif des Grandes Rousses), vallée Blanche et Argentières en 1980, ont permis de préciser le cheminement de l'eau de la surface au lit sous-glaciaire et son rôle dans le glissement de ces glaciers dits tempérés. Après son hivernage à la station Charcot (Antarctique de l'Est) et dans le cadre de la troisième année polaire internationale 1957-58, Claude Lorius participe activement à un raid d'exploration organisé par les américains en Antarctique dans le cadre de l'Année géophysique internationale (AGI). L'idée de reconstruire le climat et la composition de l'atmosphère sur plusieurs milliers d'années à partir de l'analyse des glaces polaires a probablement fait son chemin durant ce raid de plus de 100 jours. Un premier carottage de 900 mètres en 1978 à la station de Concordia permet de reconstruire le climat sur plus de 40000 ans. Mais, c'est encore au temps de la guerre froide que C. Lorius obtient en 1984 le support de la National Science Foundation (USA) et l'accord de l'Académie des sciences de Russie et de l'Arctic and Antarctic Research Institute de SaintPétersbourg pour l'analyse de la carotte de Vostok (Antarctique de l'Est) couvrant plus de 150000 ans, soit l'ensemble du dernier cycle climatique. Le $1^{\text {er }}$ octobre 1987, trois articles sont publiés dans la revue Nature montrant l'évolution du climat et de la teneur en gaz carbonique et méthane. Ce journal emploie le terme de " corne d'abondance ».

5 Le forçage climatique du $\mathrm{CO}_{2}$ était mis en évidence. Les carottages réalisés toujours à Vostok et récemment à Concordia sur plus de $3000 \mathrm{~m}$ permettent d'appréhender le changement $\mathrm{du}$ rythme glaciaire/interglaciaire sur plus de 800000 ans. La compréhension des relations de phase entre forçages et réponses climatiques se précise. Ces informations sont déjà largement utilisées pour améliorer les modèles de climat. D'autres résultats probablement moins connus méritent d'être évoqués :

- la diminution importante du volume des glaciers observée actuellement est en grande partie provoquée par une augmentation de la fusion, estivale pour les Alpes et toute l'année pour les Andes. En revanche, la phase d'avancée des glaciers entre 1760 et 1830 (paroxysme du « Petit Âge de Glace ») serait surtout la conséquence de plus fortes précipitations hivernales ;

- l'augmentation récente de la fonte de la glace du Groenland avec ses conséquences sur le niveau des mers a pu être simulée par un modèle climatique dit régional. En Antarctique, les 
modèles de climat prévoient une augmentation des précipitations et toujours une faible fonte côtière. Le devenir de la calotte antarctique dépend essentiellement de la stabilité de son écoulement ;

- des données originales, notamment à l'observatoire Vallot (massif du Mont-Blanc) ont permis de montrer l'importance des émissions anthropiques de composés organiques tant à l'échelle locale des vallées alpines qu'à l'échelle européenne pour le mont Blanc ;

- sur l'île du Spitzberg (Arctique), des teneurs relativement élevées de mercure ont été trouvées dans la neige. Il s'agit maintenant d'évaluer l'afflux de mercure dans les écosystèmes durant la période de fonte de la neige ;

- le paradigme d'un écoulement viscoplastique régulier et homogène est mis à mal pour un grand nombre de matériaux et ceci grâce en partie aux mesures effectuées au LGGE. Le cristal de glace se déforme par des mouvements collectifs de dislocations associés à des avalanches de dislocations et non par le glissement continu de ces défauts cristallins comme attendu.

6 La réputation du LGGE peut aussi se percevoir au travers de ses nombreux prix et distinctions nationales et internationales. Nous pouvons citer la médaille d'or du CNRS obtenue en 2002 par Claude Lorius (LGGE) et Jean Jouzel (LSCE).

Le LGGE et l'Année polaire internationale 2007-2008

7 La communauté scientifique internationale se rassemble en 2008 pour une 4e année polaire internationale (API, 2007-2008) afin de permettre une avancée rapide des connaissances sur ces régions polaires. Il s'agit aussi de répondre aux questions que tous se posent actuellement sur l'évolution de notre environnement. Les milieux polaires sont, en effet, des témoins privilégiés des évolutions environnementales et climatiques à l'échelle de la planète.

8 Dans le cadre de cette API, le LGGE est impliqué dans plusieurs projets internationaux. Il va intensifier ses actions de communication destinées au public : scolaires, étudiants, enseignants et media. Il participe ainsi activement à la mise en place d'une manifestation événementielle à Grenoble, centrée sur l'année polaire et pilotée par le CCSTI de Grenoble, le CNRS Alpes, l'université Joseph-Fourier, l'OSUG et le Muséum d'histoire naturelle.

9 D’avril à septembre 2008, des expositions, conférences, animations, visites de laboratoires seront organisées. La célébration des 50 ans du LGGE associée à cette API est donc l'occasion de sensibiliser le public aux recherches menées dans les régions polaires ainsi qu'aux variations du volume des glaces des glaciers de haute et basse latitude en relation avec le niveau des mers. Plus généralement, le laboratoire se doit d'organiser la réflexion autour des problématiques soulevées par les changements environnementaux. C'est donc un rendez-vous tourné vers l'avenir avec des objectifs multiples, d'une recherche fondamentale compétitive à un transfert objectif des résultats destinés aux décideurs et au public.

Le LGGE aujourd'hui

Le LGGE est maintenant une unité mixte de recherche CNRS/UJF-Grenoble et est rattaché à l'Observatoire des sciences de l'Univers de Grenoble (OSUG). Au CNRS, Le LGGE dépend principalement du département « Mathématiques, physique, planète et Univers » (MPPU), mais également du département «Sciences et technologies de l'information et de l'ingénierie » (ST2I). Il regroupe plus de quarante chercheurs CNRS et enseignants-chercheurs, une vingtaine de techniciens et ingénieurs et plus de vingt doctorants. Il bénéficie largement du support technique de l'institut polaire Paul- 
Émile-Victor (IPEV) pour ses opérations dans les régions polaires. Le LGGE conduit ses recherches en région polaire en étroite collaboration avec le Laboratoire des sciences du climat et de l'environnement (LSCE) de l'institut Pierre-Simon Laplace de Paris. Le laboratoire participe à l'enseignement dans plusieurs unités de formation de l'université Joseph-Fourier de Grenoble et joue un rôle fondamental de diffusion des connaissances via des conférences et des articles publiés dans des revues internationales, des interventions en milieu universitaire, scolaire et "grand public ». La célébration des cinquante ans du LGGE se conjugue avec le $125^{\mathrm{e}}$ anniversaire de la première Année polaire internationale (API).

11 Louis Lliboutry nous a quittés en 2007 à l'âge de 85 ans. La communauté glaciologique lui est redevable de l'impulsion extraordinaire qu'il a donnée à cette discipline, de cette œuvre considérable ainsi que de son exigence de qualité et de rigueur. Outre ses nombreuses publications, Louis Lliboutry est l'auteur de plusieurs ouvrages, de la glaciologie à la géophysique. Les 50 ans du LGGE nous donnent l'occasion de mettre en avant la place qu'il a occupée dans le développement de ce laboratoire.

\section{RÉSUMÉS}

Créé en 1958 par Louis Lliboutry, le Laboratoire de glaciologie et géophysique de l'environnement (LGGE) a bâti sa renommée scientifique sur l'étude du climat et de la composition de l'atmosphère au travers d'archives que constituent la neige et la glace accumulées au cours du temps sur les glaciers et calottes polaires. Paul Duval et Véronique Roux reviennent sur cette institution cinquantenaire.

Created by Louis Lliboutry in 1958, LGGE has built its scientific reputation on its research dealing with the past climate and the composition of the atmosphere. The various fields of research at LGGE are presented by Paul Duval and Véronique Roux.

\section{INDEX}

Mots-clés : environnement, climat, glaciologie, Glaciers, calottes polaires, LGGE, Laboratoire de glaciologie et géophysique de l'environnement (LGGE)

\section{AUTEURS}

\section{PAUL DUVAL}

Paul Duval, directeur de recherche au CNRS, est membre du Laboratoire de glaciologie et géophysique de l'environnement (LGGE).

\section{VÉRONIQUE ROUX}

Véronique Roux, personnel ITA, chargée de la communication et de la documentation, est membre du Laboratoire de glaciologie et géophysique de l'environnement (LGGE). 